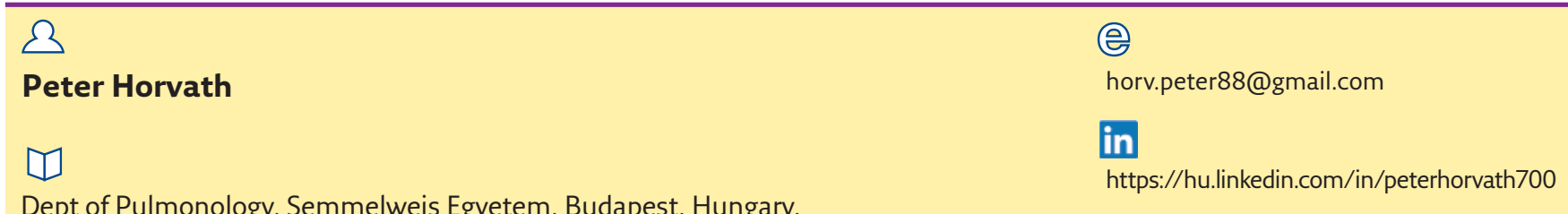

Dept of Pulmonology, Semmelweis Egyetem, Budapest, Hungary.

https://hu.linkedin.com/in/peterhorvath700

\title{
My night on call in Hungary
}

When I agreed to contribute a short piece about my experiences as a junior doctor in respiratory medicine I was spending the night on call at my workplace, the Department of Pulmonology, Semmelweis University. So it seemed pretty straightforward to write about my very recent journey through that night, including details to give you a general overview of my nights spent on call. I decided to write this essay the next morning, to ensure I would still be in the same mood.

In my department the night shift starts at 16:00 h on weekdays. There are two doctors on call every day, a respiratory medicine specialist and a junior doctor. We have four wards, three of which belong to the general pulmonology department and one of which is the noninvasive ventilation unit that is essentially a sub-intensive care unit. As the junior doctor you are responsible for two wards in the general pulmonology department, the remaining wards are covered by the specialty doctor. Nights on call differ every time. Sometimes you have an easy shift without too many new patients and with stable inpatients (the rare case), whereas sometimes you have a lot on your hands (the not so rare case).

In Hungary we operate an alert system for hospitals, which means that certain hospitals are responsible for some special tasks on different days of the week (at my workplace we are responsible for the treatment of homeless people with respiratory diseases on Mondays, treatment of pneumothoraxes on Wednesdays and bronchology on Sundays). Days on which our department is assigned a specific task usually result in an increase in patient influx.

I usually use the doctor's room in ward D as my base during my night on call. In this room I can review radiographic images in high quality and also have access to the electronic patient registration system. This room is also ideal because of its proximity to every ward.

The reception of patients happens at the entrance of the department. Our hospital has different buildings for the different departments, and to date, there is no emergency department to sift the patients. For this reason, we quite often receive people with cardiac disease, as the leading symptom is dyspnoea which often misleads the first line healthcare provider (either a general practitioner or the ambulance staff). Unfortunately, there are no administrative staff or auxiliary assistance, therefore we have to do the paperwork ourselves and we also take the $X$-rays. If a patient needs to be admitted to hospital, and they usually do, then all these tasks take up a substantial amount of time.

The usual admission procedure for a patient is as follows.

1) An ambulance arrives and the doorman calls you.

2) You go downstairs and listen to the referral by the ambulance staff. Following a quick examination, if the patient seems to be stable and to have a respiratory disease, start the administration. If the patient is unstable, carry out an arterial blood gas (ABG) analysis, if this supports your initial hunch, call the specialty doctor.

3) If the patient is admitted to the department of general pulmonology we carry out a radiograph, ABG analysis and run blood tests.

4) Start therapy.

5) Follow-up the patient.

Nights on call differ from day-to-day. I think it is a similarity among the different countries. The last night on call I worked was on the verge of average and difficult. We had seven patients: two pulmonary embolisms, two asthma exacerbations, one
Cite as: Horvath P. My night on call in Hungary. Breathe 2016; 12: 287-288. 


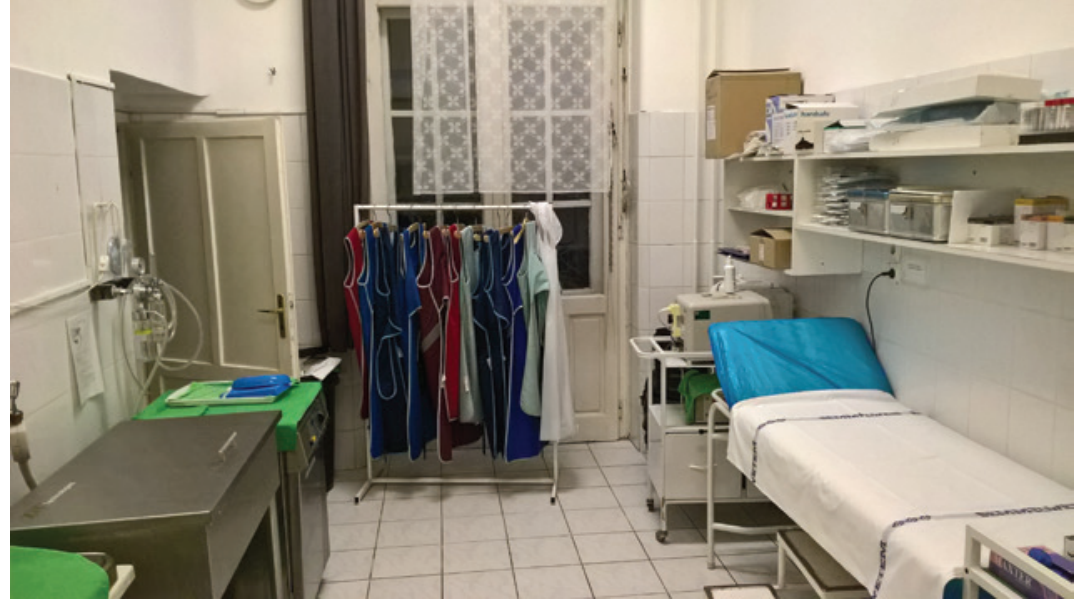

Figure 1 This is one of the rooms in our bronchology unit. We perform the local anaesthesia before bronchoscopies here and this is where invasive procedures take place (e.g. thoracenteses, fine-needle aspiration biopsies or tube thoracostomies).

lung transplant patient, one patient with suspected tuberculosis and one patient with lung cancer.

I prefer the nights where we have a patient who requires an invasive procedure, like thoracostomy or bronchoscopy. Most specialty doctors let you carry out the procedure and they assist you. During this recent night on call I was lucky, I had the chance to do an ultrasound guided thoracentesis on the lung cancer patient due to recurrent pleural fluid (figure 1).
If your night is not so busy, you also have plenty of time to practice. We have a dummy doll on which you can do various procedures like endotracheal intubation or bronchoscopy. The clinic also has three ultrasound machines, which are very useful to practice. During my training I have different procedures that I need to master: I started with bronchoscopies for the first few months), then I put some emphasis on basic ultrasonography (examination of the lungs, pleural cavity and fast examinations). When I did my cardiology rota I spent my free time performing transthoracic echocardiography, which I find extremely useful for the differential diagnosis of dyspnoea. Currently I am practicing endotracheal intubation (figure 2).

All in all, I would say that nights on call are a difficult, tiring, but also very interesting and edifying part of our training. You have an opportunity to practice your knowledge and manual skills in real life scenarios under supervision. Despite sometimes feeling I have had enough of them, deep inside I think like these long nights.

What is also a very important part of these occasions is that I give myself some treat the next day to pat myself on the head for my performance. I usually have breakfast in an Italian diner on my way home on Oktogon, which is a plaza in the centre of Budapest. However, this time, as summer is coming, I decided to have a nice morning at one of the most popular beaches in Budapest, Palatinus.

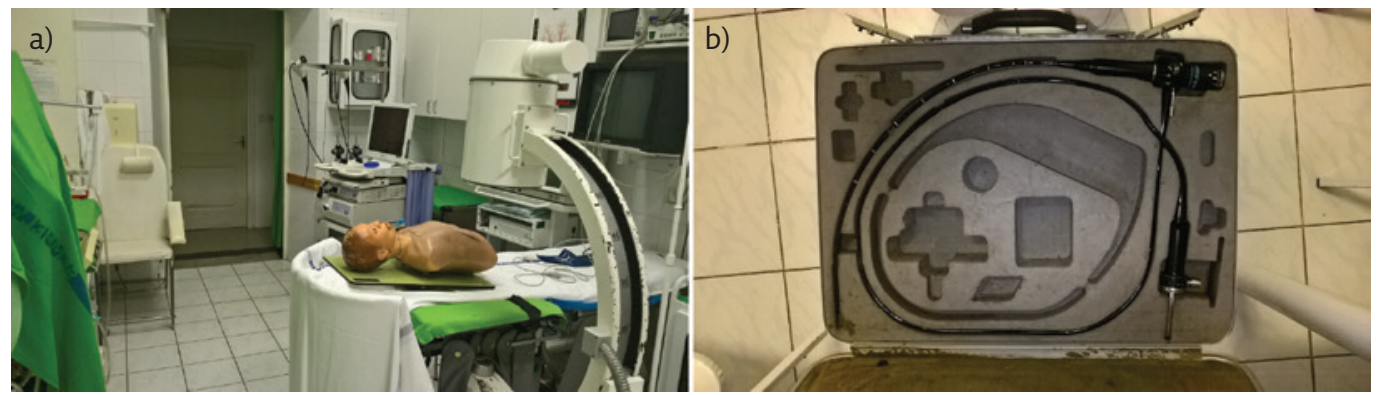

Figure 2 a) A view of the bronchoscopy suite with our dummy doll. Here you can see it after a successful endotracheal intubation. b) A picture of the bronchoscope we use to practice.

\section{Conflict of interest}

None declared. 